Preprint typeset in JHEP style - HYPER VERSION

CERN-PH-TH/2009-127

MPP-2009-81

\title{
Flavour-dependent radiative correction to neutrino-neutrino refraction
}

\author{
Alessandro Mirizzi ${ }^{1}$, Stefano Pozzorini ${ }^{2}$, Georg G. Raffelt ${ }^{1}$, Pasquale D. Serpico ${ }^{2}$ \\ ${ }^{1}$ Max-Planck-Institut für Physik (Werner-Heisenberg-Institut), Föhringer Ring 6, \\ 80805 Munich, Germany \\ ${ }^{2}$ Physics Department, Theory Group, CERN, CH-1211 Geneva 23, Switzerland \\ E-mails: amirizzi@mppmu.mpg.de, stefano.pozzorini@cern.ch, \\ raffelt@mppmu.mpg.de, serpico@cern.ch
}

\begin{abstract}
In the framework of the Standard Model we calculate the flavour non-universal correction for neutrino refraction in a neutrino background and verify a similar previous result for the case of ordinary-matter background. The dominant term arises at loop level and involves $\tau$ leptons circulating in the loop. These $\mathcal{O}\left(G_{\mathrm{F}} m_{\tau}^{2}\right)$ corrections to the tree-level potential provide the dominant refractive difference between $\nu_{\mu}$ and $\nu_{\tau}$ unless the medium contains $\mu$ or $\tau$ leptons. Our results affect the flavour evolution of dense neutrino gases and may be of interest for collective three-flavour oscillations of supernova neutrinos. We spell out explicitly how these non-universal neutrino-neutrino interactions enter the flavour oscillation equations.
\end{abstract}

Keywords: Neutrino Physics, Standard Model, Supernovae. 


\section{Contents}

1. Introduction 1

2. Calculation of the radiative corrections 3

2.1 Tree level 3

2.2 Radiative correction for a background of charged fermions 5

2.3 Extension to neutrinos as background fermions 8

3. Including radiative corrections in the oscillation equations 11

4. Conclusions 12

\section{Introduction}

The dispersion relation of any particle is modified by a background field or medium. For neutrinos this effect is extremely faint, yet the matter effect is crucial for neutrino oscillations where the feeble refractive indices compete with the minuscule mass differences [1, 2].

Since neutral-current interactions among the three active neutrinos are flavour universal, they can be usually ignored for neutrino oscillation phenomenology, where only the relative energy shift between flavours is important. Moreover, energy scales of interest are well below the weak boson mass $M_{W}$, so for low-energy neutrinos in ordinary matter (no $\mu$ or $\tau$ leptons) the potential-energy shift between flavours can be calculated simply from the effective charged-current Hamiltonian density

$$
\mathcal{H}_{\mathrm{MSW}}=\frac{G_{\mathrm{F}}}{\sqrt{2}}\left[\bar{e} \gamma_{\alpha}\left(1-\gamma_{5}\right) \nu_{e}\right]\left[\bar{\nu}_{e} \gamma^{\alpha}\left(1-\gamma_{5}\right) e\right]=\frac{G_{\mathrm{F}}}{\sqrt{2}}\left[\bar{\nu}_{e} \gamma_{\alpha}\left(1-\gamma_{5}\right) \nu_{e}\right]\left[\bar{e} \gamma^{\alpha}\left(1-\gamma_{5}\right) e\right]
$$

where the second equality follows from the usual Fierz rearrangement. One thus finds the well-known tree-level energy splitting for electron neutrinos with respect to $\nu_{\mu}$ or $\nu_{\tau}$,

$$
\Delta E_{\nu_{e} \nu_{\mu, \tau}}=E_{\nu_{e}}-E_{\nu_{\mu, \tau}}=\sqrt{2} G_{\mathrm{F}} n_{e}
$$

where $n_{e}$ is understood as net density of electrons minus positrons. In lowest order of perturbation theory, the $\nu_{\mu}$ and $\nu_{\tau}$ dispersion relations coincide and $\Delta E_{\nu_{\mu} \nu_{\tau}}=0$.

Equation (1.2) provides a sufficient description of the matter effect in most practical cases, most notably solar neutrinos, but care must be taken in some circumstances where either the leading term vanishes $\left(n_{e} \simeq 0\right)$ or subtle three-flavour effects come into play. For example, it has long been appreciated that radiative corrections break the $\nu_{\mu}-\nu_{\tau}$ degeneracy in an ordinary medium because the charged-lepton mass $\left(m_{\mu}\right.$ or $\left.m_{\tau}\right)$ in the loops distinguishes between flavours [3]. In the low-energy limit, the loop-induced $\nu_{\mu}-\nu_{\tau}$ energy 
shift has the same structure as Eq. (1.2) and thus can be represented by an effective net tau-lepton density in the form

$$
\Delta E_{\nu_{\tau} \nu_{\mu}}=E_{\nu_{\tau}}-E_{\nu_{\mu}}=\sqrt{2} G_{\mathrm{F}} n_{\tau}^{\mathrm{eff}} .
$$

Botella, Lim and Marciano [3] studied explicitly the case of an ordinary electrically neutral medium and found the equivalent of

$$
n_{\tau}^{\mathrm{eff}}=\epsilon \frac{3}{2}\left[\log \left(\frac{M_{W}^{2}}{m_{\tau}^{2}}\right)-1+\frac{Y_{n}}{3}\right] n_{B},
$$

where $n_{B}=n_{p}+n_{n}$ is the baryon density and $Y_{n}=n_{n} / n_{B}$ the neutron/baryon fraction, the remainder being protons. We have introduced the small parameter

$$
\epsilon=\frac{G_{\mathrm{F}} m_{\tau}^{2}}{\sqrt{2} \pi^{2}}=2.64 \times 10^{-6}
$$

that will appear in all of our results. Here and in the following we neglect $m_{e}, m_{\mu}$, the first-generation quark masses and the neutrino energy relative to $m_{\tau}$ and $M_{W}$.

The quantity $Y_{n} / 3$ in Eq. (1.4) is at most a $5 \%$ correction. Neglecting it provides

$$
n_{\tau}^{\mathrm{eff}} \simeq 2.6 \times 10^{-5} n_{B} .
$$

The only known example where this small density can be of practical interest is the case of supernova $(\mathrm{SN})$ neutrinos propagating in a dense environment after leaving the neutrino sphere of the collapsed core. At large distances they encounter two resonances where the usual matter effect causes a level crossing with the "atmospheric" and "solar" mass differences, respectively [4]. Much closer to the core the radiative effect will cause similar resonances [5]. However, the $\nu_{\mu}, \bar{\nu}_{\mu}, \nu_{\tau}$ and $\bar{\nu}_{\tau}$ fluxes and spectra are probably equal, so one may think that $\nu_{\mu}-\nu_{\tau}$ transformations play no role.

This picture has radically changed with the insight that typically flavour oscillations do occur close to the neutrino sphere. The refractive effect of neutrinos on neutrinos is not fully captured by a refractive energy shift because if neutrinos oscillate, the same applies to the neutrino background, providing a "flavour off-diagonal refractive index" [6, 7]. In other words, the neutrino oscillation equations become intrinsically nonlinear, leading to collective forms of oscillation [8, 9], a subject of intense recent investigation (see the recent review [10] and references therein). Since collective oscillations operate close to the neutrino sphere, here the $\nu_{\mu}-\nu_{\tau}$ resonance can become important in a three-flavour treatment 11, 12]. On the other hand, collective oscillations are suppressed if the matter effect is too large, typically when $n_{B} \gtrsim n_{\nu}$ [13]. The SN matter profile and neutrino flux vary with time and depend strongly on the properties of the progenitor star, so depending on circumstances $n_{\nu}$ can be larger or smaller than $n_{B}$ in the collective oscillation region a few hundred kilometers above the neutrino sphere. When $n_{\nu} \gtrsim n_{B}$ and non-linear effects become important, it is reasonable to expect that the leading effect breaking the degeneracy between $\nu_{\mu}$ and $\nu_{\tau}$ is actually provided by loop-induced effects due to the neutrino background itself. In this article we fill a gap in the literature and calculate this non-universal radiative correction for neutrino refraction in a neutrino background. 
Our calculation is described in Sec. 2 where we begin with a background of charged fermions, confirming the original result shown in Eq. (1.4). From this starting point we include neutrinos as a background and derive our main result, an effective neutrino-neutrino Hamiltonian that includes $m_{\tau}$ effects in the loop. In Sec. 3 we spell out how to include our result in the non-linear flavour oscillation equations before concluding in Sec. 1 .

\section{Calculation of the radiative corrections}

The energy shift between different neutrino species in a given medium is proportional to the difference of forward scattering amplitudes [14, 15]. Since we deal with energies well below the electroweak scale, the results of the one-loop calculation within the electroweak theory can be expressed in terms of an effective four-fermion Hamiltonian. Generically, the quantum effects manifest themselves in the low-energy theory as corrections to the coefficients of the operators. (In addition they could induce higher-order operators mediating processes forbidden in the original theory.) The corrections, however, need not respect the original symmetries of the interaction. The case at hand makes no exception: we shall see by explicit calculation that at one loop, although the Lorentz structure of the treelevel current-current interaction is preserved, flavour universality of the "neutral currents" breaks down.

\subsection{Tree level}

To set up our problem and fix the notation, we recall that neutrinos interact with matter via the charged and neutral weak-current Lagrangian densities

$$
\begin{aligned}
& \mathcal{L}_{\mathrm{CC}}=-\frac{g}{2 \sqrt{2}}\left(J_{\alpha}^{\mathrm{CC}} W^{+\alpha}+J_{\alpha}^{\mathrm{CC}}{ }^{\dagger} W^{-\alpha}\right), \\
& \mathcal{L}_{\mathrm{NC}}=-\frac{g}{2 c_{\mathrm{w}}} J_{\alpha}^{\mathrm{NC}} Z^{\alpha} .
\end{aligned}
$$

Here, $g$ denotes the $\mathrm{SU}(2)$ weak coupling, $c_{\mathrm{w}}=\sqrt{1-s_{\mathrm{w}}^{2}}$ is the cosine of the weak mixing angle, and $W^{ \pm}$and $Z$ represent the charged and neutral gauge bosons with masses $M_{W}$ and $M_{Z}=M_{W} / c_{\mathrm{w}}$, respectively. These parameters are related to the Fermi constant $G_{\mathrm{F}}$ and the fine structure constant $\alpha$ by

$$
G_{\mathrm{F}}=\frac{\sqrt{2} g^{2}}{8 M_{W}^{2}}=\frac{\pi \alpha}{\sqrt{2} s_{\mathrm{w}}^{2} M_{W}^{2}} .
$$

In terms of the weak-interaction eigenstates, the charged current is

$$
J_{\alpha}^{\mathrm{CC}}=\sum_{l} \bar{\nu}_{l} \gamma_{\alpha}\left(1-\gamma_{5}\right) l+\sum_{i} \bar{u}_{i} \gamma_{\alpha}\left(1-\gamma_{5}\right) d_{i}
$$

while the neutral current is

$$
\begin{aligned}
J_{\alpha}^{\mathrm{NC}} & =\sum_{l}\left[\bar{\nu}_{l} \gamma_{\alpha}\left(c_{V}^{\nu}-c_{A}^{\nu} \gamma_{5}\right) \nu_{l}+\bar{l} \gamma_{\alpha}\left(c_{V}^{l}-c_{A}^{l} \gamma_{5}\right) l\right]+ \\
& +\sum_{i}\left[\bar{u}_{i} \gamma_{\alpha}\left(c_{V}^{u}-c_{A}^{u} \gamma_{5}\right) u_{i}+\bar{d}_{i} \gamma_{\alpha}\left(c_{V}^{d}-c_{A}^{d} \gamma_{5}\right) d_{i}\right] .
\end{aligned}
$$


The indices $l$ and $i$ run over the lepton and quark generations, respectively. The vector and axial couplings of a fermion $f=\nu, e, u$ or $d$ are

$$
c_{V}^{f}=T_{3 f}-2 Q_{f} s_{\mathrm{w}}^{2}, \quad c_{A}^{f}=T_{3 f},
$$

where $Q_{f}=\left\{0,-1, \frac{2}{3},-\frac{1}{3}\right\}$ is the electric charge and $T_{3 f}=\left\{\frac{1}{2},-\frac{1}{2}, \frac{1}{2},-\frac{1}{2}\right\}$ is the third component of the weak isospin. In particular $c_{V}^{\nu}=c_{A}^{\nu}=1 / 2$ for neutrinos. We need to calculate the shift in the potential of a neutrino $\nu_{l}=\nu_{\mu}, \nu_{\tau}$ interacting with a medium consisting of ordinary matter, i.e. fermions $f=u, d, e$. This interaction is described by the effective neutral-current Hamiltonian density

$$
\mathcal{H}_{\nu_{l} f}=\frac{G_{\mathrm{F}}}{\sqrt{2}}\left[\bar{\nu}_{l} \gamma_{\alpha}\left(1-\gamma_{5}\right) \nu_{l}\right]\left[\bar{f} \gamma^{\alpha}\left(c_{V}^{f}-c_{A}^{f} \gamma_{5}\right) f\right] .
$$

Charged-current interactions can be ignored since we consider only the potential-energy difference between $\mu^{-}$and $\tau$-neutrinos, which is a purely radiative effect, and the medium is free from $\mu$ - and $\tau$-leptons.

Assuming homogeneity, the potential in terms of the Dirac spinors of the (left-handed) neutrino $u_{\nu}(p)$ and of the fermion $u_{f}(k, s)$ is

$$
V_{\nu_{l} f}=\frac{G_{\mathrm{F}}}{\sqrt{2}} \sum_{s} \int \mathrm{d}^{3} \mathbf{k} \mathrm{d}^{3} \mathbf{x} F_{f}(\mathbf{k}, s)\left[\bar{u}_{\nu}(p) \gamma_{\alpha}\left(1-\gamma_{5}\right) u_{\nu}(p)\right]\left[\bar{u}_{f}(k, s) \gamma^{\alpha}\left(c_{V}^{f}-c_{A}^{f} \gamma_{5}\right) u_{f}(k, s)\right]
$$

where the spatial integral is over the normalization volume (containing one neutrino), $F_{f}(\mathbf{k}, s)$ is the momentum distribution of the fermion background (normalized to 1), and $s$ denotes the fermion polarization. The matrix elements are calculated between identical initial and final states, both in momentum and spin, i.e. they correspond to the forward scattering amplitude. This leads to [16]

$$
\begin{aligned}
V_{\nu_{l} f} & =\frac{G_{\mathrm{F}}}{\sqrt{2}} \sum_{s} \int \mathrm{d}^{3} \mathbf{k} F_{f}(\mathbf{k}, s)\left(\frac{2 p_{\nu}^{\alpha}}{E_{\nu}}\right)\left[\bar{u}_{f}(k, s) \gamma_{\alpha}\left(c_{V}^{f}-c_{A}^{f} \gamma_{5}\right) u_{f}(k, s)\right] \\
& =\sqrt{2} G_{\mathrm{F}} \sum_{s} \int \mathrm{d}^{3} \mathbf{k} F_{f}(\mathbf{k}, s)\left(\frac{p_{\nu}^{\alpha}}{E_{\nu}}\right)\left(n_{f} \frac{c_{V}^{f} k_{\alpha}-c_{A}^{f} m_{f} s_{\alpha}}{E_{f}}\right),
\end{aligned}
$$

where $m_{f}$ is the background fermion mass and $s^{\alpha}$ its polarization four vector, such that $s \cdot k=0, s^{2}=-1$.

When the medium consists of unpolarized ordinary-matter fermions $(f=e, u, d)$, the term linear in $s^{\alpha}$ averages to zero. Thus the neutrino potential is independent of the axial coupling $c_{A}^{f}$ and reads

$$
V_{\nu_{l} f}=\sqrt{2} G_{\mathrm{F}} n_{f} c_{V}^{f} \sum_{s} \int \mathrm{d}^{3} \mathbf{k} F_{f}(\mathbf{k}, s)\left(1-\mathbf{v}_{\mathbf{p}} \cdot \mathbf{v}_{\mathbf{k}}\right)=\sqrt{2} G_{\mathrm{F}} n_{f} c_{V}^{f},
$$

where $\mathbf{v}_{\mathbf{p}}$ and $\mathbf{v}_{\mathbf{k}}$ are the neutrino and fermion velocities, with momentum $\mathbf{p}$ and $\mathbf{k}$, respectively. In the last equality we also assumed isotropy of the background distribution.

As far as the tree-level neutral-current interaction in Eq. (2.7) is concerned, $\nu_{\mu}, \nu_{\tau}$, and $\nu_{e}$ receive a common potential shift described by the flavour-independent result of Eq. 2.10), which is irrelevant for neutrino oscillations. Note also that, in a neutral medium, the sum over all species cancels the terms proportional to the electric charge $Q_{f}$ in Eq. (2.6). 


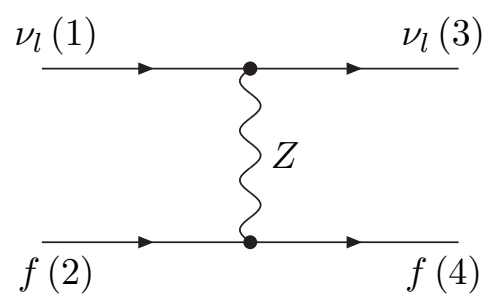

Figure 1: At tree level, neutrino forward scattering in a charged-fermion background $(f=u, d, e)$ proceeds through $t$-channel exchange of $Z$ bosons.

\subsection{Radiative correction for a background of charged fermions}

The effect we are interested in is the flavour-dependent part of the loop contribution to the neutral-current couplings $c_{V / A}^{f}$ in Eq. (2.7). Technically, the matching of the effective Hamiltonian coefficients will be obtained by computing the $\nu_{l}-f$ forward scattering amplitude. Note also that the momenta of the scattering particles will be set to zero, since their typical values in SNe are very small compared to the relevant mass scales $m_{\tau}$ and $M_{W}$. In this approximation all one-loop integrals reduce to tadpoles.

At tree level, $\nu_{l}$ forward scattering in a charged-fermion background is described by the Feynman diagram in Fig. 1. The corresponding amplitude is

$$
\mathcal{A}_{1}^{\nu_{l} f}=\frac{-i g^{2}}{4 M_{W}^{2}}\left[\bar{u}_{3} \gamma^{\mu} \omega_{L} u_{1}\right]\left[\bar{u}_{4} \gamma_{\mu}\left(c_{V}^{f}-c_{A}^{f} \gamma_{5}\right) u_{2}\right]=\frac{-i g^{2}}{4 M_{W}^{2}} \mathcal{W}_{31} \mathcal{C}_{42}^{f},
$$

where $\omega_{L}=\left(1-\gamma_{5}\right) / 2$ is the left-handed projector and we have introduced the currents

$$
\mathcal{C}_{i j}^{f}=\bar{u}_{i} \gamma_{\mu}\left(c_{V}^{f}-c_{A}^{f} \gamma_{5}\right) u_{j}, \quad \mathcal{W}_{i j}=\mathcal{C}_{i j}^{\nu_{l}}=\bar{u}_{i} \gamma^{\mu} \omega_{L} u_{j}
$$

where the Lorentz indices and their contraction are implicitly understood. At one loop, neutrino-flavour dependent contributions result only from diagrams where a charged gauge boson $\left(W^{ \pm}\right)$or would-be-Goldstone boson $\left(\phi^{ \pm}\right)$couples to a neutrino line, giving rise to a virtual lepton. In practice we will restrict ourselves to this type of diagrams and we will retain only terms that depend on the mass of the lepton and are thus sensitive to its flavour. Flavour-independent contributions will be systematically neglected. Note that here and throughout we work in the 't Hooft-Feynman gauge, in which $M_{\phi}=M_{W}$.

As we will see, it is convenient to classify the loop diagrams according to their topology and to treat box and non-box diagrams separately. The latter represent the corrections to the $\nu_{l} \bar{\nu}_{l} Z$ vertex and comprise the vertex diagrams of Figs. $2-3$ as well as the selfenergy insertions of Fig. 1 . Note that all diagrams of non-box type are independent of the nature of the background fermions $f$ in the sense that the tree-level $f \bar{f} Z$ vertex factorizes. Box diagrams can involve two charged bosons $(W W, W \phi$ or $\phi \phi)$ and are suppressed unless both of them are $W$ bosons. Depending on the isospin nature of the background fermions,

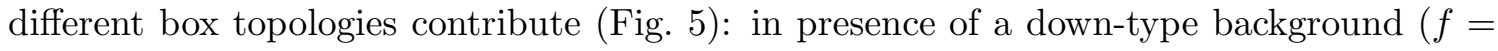
$e, d)$ only ladder boxes appear, while crossed boxes contribute only if the background fermions are of up type $(f=u$ and, for later application, also $f=\nu)$. 

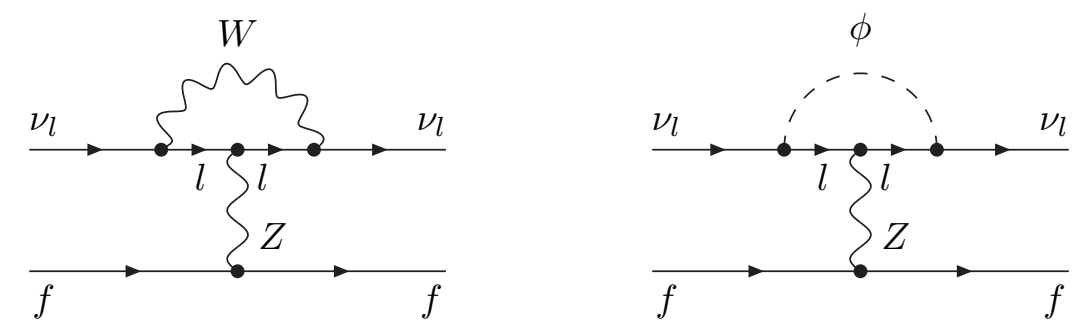

Figure 2: Flavour-dependent vertex corrections involving $Z l \bar{l}$ interactions.
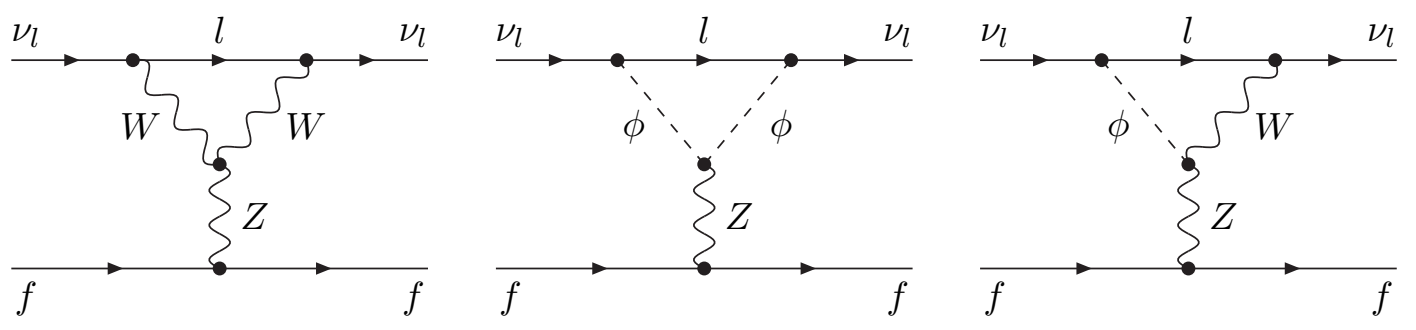

Figure 3: Flavour-dependent vertex corrections involving $Z W^{+} W^{-}, Z \phi^{+} \phi^{-}$, and $Z W^{+} \phi^{-}$interactions. In the latter case (rightmost diagram), an additional contribution with $W \leftrightarrow \phi$ must be taken into account.
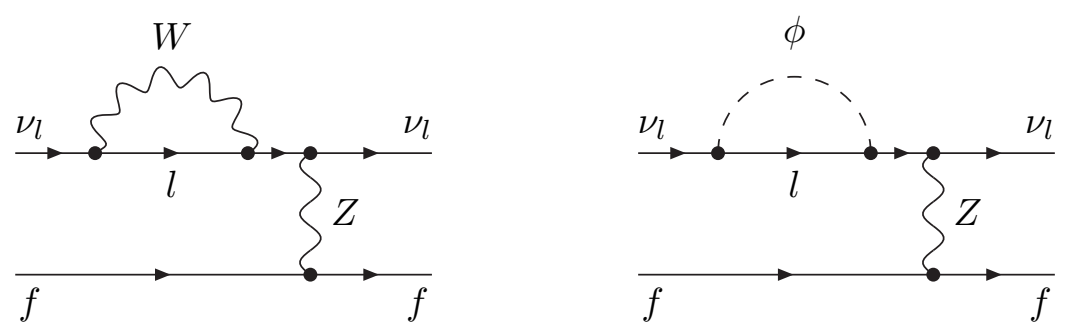

Figure 4: Flavour-dependent selfenergy insertions in the incoming neutrino line. Analogous diagrams for the outgoing neutrino must be considered. These diagrams have to be understood as on-shell renormalization factors for the neutrino wave functions.

Apart from flavour-independent additive terms, the relevant amplitudes for the diagrams in Fig. 2 are

$$
\begin{aligned}
& \mathcal{A}_{2, W}^{\nu_{l} f}=+\frac{i g^{4}}{4(4 \pi)^{2}} \frac{m_{l}^{2}}{M_{W}^{4}}\left[\frac{1}{2}+s_{\mathrm{w}}^{2}+\log \frac{m_{l}^{2}}{M_{W}^{2}}\right] \mathcal{W}_{31} \mathcal{C}_{42}^{f}, \\
& \mathcal{A}_{2, \phi}^{\nu_{l} f}=-\frac{i g^{4}}{4(4 \pi)^{2}} \frac{m_{l}^{2}}{M_{W}^{4}} \frac{s_{\mathrm{w}}^{2}}{2}\left[\Delta+\frac{1}{2}+\log \frac{\mu^{2}}{M_{W}^{2}}\right] \mathcal{W}_{31} \mathcal{C}_{42}^{f} .
\end{aligned}
$$



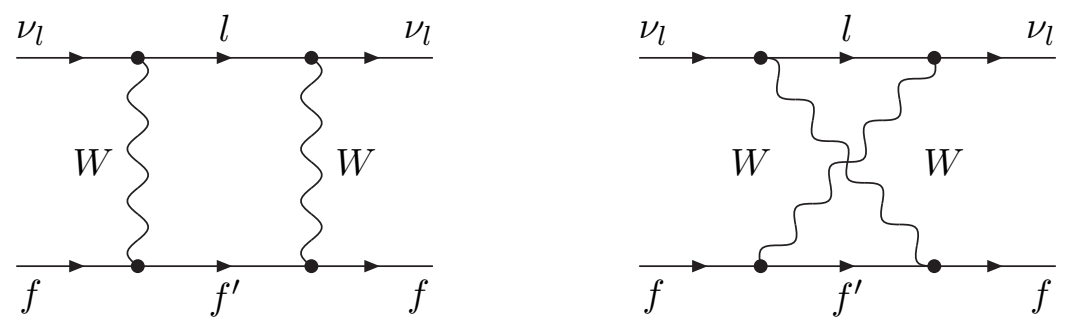

Figure 5: Flavour-dependent box corrections. The ladder box diagrams (left) contribute only for down-type $(f=e, d)$ background fermions, whereas the crossed box diagrams (right) contribute only for up-type $(f=u, \nu)$ fermions. Box diagrams involving $\phi^{ \pm}$exchange are suppressed.

For the diagrams in Fig. 3 one finds

$$
\begin{aligned}
\mathcal{A}_{3, W W Z}^{\nu_{l} f} & =3 c_{\mathrm{w}}^{2} \frac{i g^{4}}{4(4 \pi)^{2}} \frac{m_{l}^{2}}{M_{W}^{4}} \mathcal{W}_{31} \mathcal{C}_{42}^{f}, \\
\mathcal{A}_{3, \phi \phi Z}^{\nu_{l} f} & =\left(s_{\mathrm{w}}^{2}-c_{\mathrm{w}}^{2}\right) \frac{i g^{4}}{4(4 \pi)^{2}} \frac{m_{l}^{2}}{M_{W}^{4}}\left(\frac{1}{8}+\frac{\Delta}{4}+\frac{1}{4} \log \frac{\mu^{2}}{M_{W}^{2}}\right) \mathcal{W}_{31} \mathcal{C}_{42}^{f}, \\
\mathcal{A}_{3, W \phi Z}^{\nu_{l} f} & =2 s_{\mathrm{w}}^{2} \frac{i g^{4}}{4(4 \pi)^{2}} \frac{m_{l}^{2}}{M_{W}^{4}} \mathcal{W}_{31} \mathcal{C}_{42}^{f},
\end{aligned}
$$

where in the last equation we included a factor two corresponding to a second diagram with $W \leftrightarrow \phi$. Finally, the relevant amplitude for the diagrams in Fig. 田 is

$$
\mathcal{A}_{4}^{\nu_{l} f}=-\frac{i g^{4}}{4(4 \pi)^{2}} \frac{m_{l}^{2}}{M_{W}^{4}}\left(\frac{3}{8}-\frac{\Delta}{4}-\frac{1}{4} \log \frac{\mu^{2}}{M_{W}^{2}}\right) \mathcal{W}_{31} \mathcal{C}_{42}^{f},
$$

where, again, we included a factor two for the correction to the second neutrino. The expression $\Delta=\frac{2}{4-D}-\gamma_{E}+\log 4 \pi$ represents ultraviolet poles within dimensional regularization and $\mu$ is the corresponding mass scale. The complete non-box contribution, i.e. the sum of the amplitudes Eqs. (2.13)-(2.18), is free from ultraviolet divergences and reads

$$
\mathcal{A}_{234}^{\nu_{l} f}=\frac{i g^{4}}{4(4 \pi)^{2}} \frac{m_{l}^{2}}{M_{W}^{4}}\left[3+\log \frac{m_{l}^{2}}{M_{W}^{2}}\right] \mathcal{W}_{31} \mathcal{C}_{42}^{f} .
$$

For the box diagrams in Fig. 5, neglecting contributions of order $m_{f^{\prime}} / M_{W}$, one obtains

$$
\begin{aligned}
& \mathcal{A}_{5}^{\nu_{l} d}=\mathcal{A}_{5}^{\nu_{l} e}=\frac{i g^{4}}{(4 \pi)^{2}} \frac{m_{l}^{2}}{M_{W}^{4}}\left[\log \left(\frac{m_{l}^{2}}{M_{W}^{2}}\right)+1\right] \mathcal{W}_{31} \mathcal{W}_{42}, \\
& \mathcal{A}_{5}^{\nu_{l} u}=-\frac{1}{4} \frac{i g^{4}}{(4 \pi)^{2}} \frac{m_{l}^{2}}{M_{W}^{4}}\left[\log \left(\frac{m_{l}^{2}}{M_{W}^{2}}\right)+1\right] \mathcal{W}_{31} \mathcal{W}_{42} .
\end{aligned}
$$

The results for down- and up-type fermions correspond to ladder and crossed box topologies, respectively.

These corrections can be described as flavour-dependent shifts,

$$
c_{V / A}^{f} \rightarrow c_{V / A}^{f}+\Delta c_{V / A}^{\nu_{l} f},
$$


of fermion couplings in the effective Hamiltonian density $\mathcal{H}_{\nu_{l} f}$ of Eq. (2.7). The shifts induced in the vector and axial couplings of down- and up-type fermions are

$$
\begin{aligned}
& \Delta c_{V / A}^{\nu_{l} d}=-\frac{g^{2}}{(4 \pi)^{2}} \frac{m_{l}^{2}}{M_{W}^{2}}\left[\left(3 c_{V / A}^{d}+2\right)+\left(c_{V / A}^{d}+2\right) \log \frac{m_{l}^{2}}{M_{W}^{2}}\right], \\
& \Delta c_{V / A}^{\nu_{l} u}=-\frac{g^{2}}{(4 \pi)^{2}} \frac{m_{l}^{2}}{M_{W}^{2}}\left[\left(3 c_{V / A}^{u}-\frac{1}{2}\right)+\left(c_{V / A}^{u}-\frac{1}{2}\right) \log \frac{m_{l}^{2}}{M_{W}^{2}}\right] .
\end{aligned}
$$

Here the terms proportional to $c_{V / A}^{f}$ originate from non-box diagrams and the remnant is due to boxes. The relevant shift for the electron component of the background is obtained by replacing $d \rightarrow e$ in Eq. (2.23).

Taking into account that in neutral, ordinary matter $n_{e}=n_{p}, n_{u}=2 n_{p}+n_{n}, n_{d}=$ $n_{p}+2 n_{n}$ and using $n_{B}=n_{p}+n_{n}$, one obtains the $\nu_{\mu}-\nu_{\tau}$ energy shift

$$
\Delta E_{\nu_{\tau} \nu_{\mu}}=\sqrt{2} G_{\mathrm{F}} \sum_{f} \Delta c_{V}^{\nu_{\tau} f} n_{f}=3 \sqrt{2} G_{\mathrm{F}} \frac{g^{2}}{(4 \pi)^{2}} \frac{m_{\tau}^{2}}{M_{W}^{2}}\left[\log \left(\frac{M_{W}^{2}}{m_{\tau}^{2}}\right)-1+\frac{Y_{n}}{3}\right] n_{B},
$$

equivalent to Eq. (1.4) and in full agreement with Ref. [3].

\subsection{Extension to neutrinos as background fermions}

Now we turn to our main case of interest, namely when the propagating neutrinos $\left(\nu_{l}\right)$ interact with a neutrino background $\left(f=\nu_{l^{\prime}}\right)$. At tree level the relevant interactions are described by the four-neutrino neutral-current effective Hamiltonian

$$
\mathcal{H}_{\nu \nu}=\sum_{l, l^{\prime}} \mathcal{H}_{\nu_{l} \nu_{l^{\prime}}}=\frac{G_{\mathrm{F}}}{\sqrt{2}} \sum_{l, l^{\prime}}\left[\bar{\nu}_{l} \gamma_{\alpha} \omega_{L} \nu_{l}\right]\left[\bar{\nu}_{l^{\prime}} \gamma^{\alpha} \omega_{L} \nu_{l^{\prime}}\right]
$$

which is of the same form as Eq. (2.7) upon identification of the fermion couplings with $c_{V}^{\nu_{l^{\prime}}}=c_{A}^{\nu_{l^{\prime}}}=1 / 2$ and noting that each term with $l \neq l^{\prime}$ enters twice in the sum. Note also that Eq. (2.26) has a $U(3)$ symmetry in flavour space.

The general form of the one-loop effective Hamiltonian is

$$
\mathcal{H}_{\nu \nu}=\sum_{l, l^{\prime}} \mathcal{H}_{\nu_{l} \nu_{l^{\prime}}}=\frac{G_{\mathrm{F}}}{\sqrt{2}} \sum_{l, l^{\prime}}\left(1+\kappa^{\nu_{l} \nu_{l^{\prime}}}\right)\left[\bar{\nu}_{l} \gamma_{\alpha} \omega_{L} \nu_{l}\right]\left[\bar{\nu}_{l^{\prime}} \gamma^{\alpha} \omega_{L} \nu_{l^{\prime}}\right]
$$

Again only flavour-dependent contributions $\kappa^{\nu_{l} \nu_{l^{\prime}}}$ are taken into account. In practice we consider only terms involving $m_{l}^{2} / M_{W}^{2}$ and/or $m_{l^{\prime}}^{2} / M_{W}^{2}$. Moreover, the electron and the muon masses are neglected, i.e. we use

$$
\frac{m_{l}^{2}}{M_{W}^{2}}=\delta_{l \tau} \frac{m_{\tau}^{2}}{M_{W}^{2}}
$$

This implies

$$
\kappa^{\nu_{e} \nu_{e}}=\kappa^{\nu_{e} \nu_{\mu}}=\kappa^{\nu_{\mu} \nu_{e}}=\kappa^{\nu_{\mu} \nu_{\mu}}=0,
$$

and one-loop terms proportional to $m_{\tau}^{2} / M_{W}^{2}$ contribute only if either $\nu_{l}$ or $\nu_{l^{\prime}}$ is a $\nu_{\tau}$.

We first consider the correction $\kappa^{\nu_{\tau} \nu_{\beta}}=\kappa^{\nu_{\beta} \nu_{\tau}}$ with $\beta \neq \tau$. This case can be easily related to the charged-fermion background results of Sec. 2.2. To this end we can regard $\nu_{\tau}$ as a neutrino that propagates in a background with $f=\nu_{\beta}$. The one-loop diagrams that give rise to terms of order $m_{\tau}^{2} / M_{W}^{2}$ are exactly the same as in Sec. 2.2: 
- In principle, in addition to the vertices and selfenergies of Figs. 214, which contribute to the $\nu_{\tau} \bar{\nu}_{\tau} Z$ vertex, diagrams corresponding to the $\nu_{\beta} \bar{\nu}_{\beta} Z$ vertex should be included. However, for $\beta \neq \tau$, such diagrams are free from $m_{\tau}^{2} / M_{W}^{2}$ contributions and can thus be neglected.

- Concerning the box diagrams of Fig. 5, the terms of order $m_{f^{\prime}}^{2} / M_{W}^{2}$ that we have neglected in Sec. 2.2 correspond to terms of order $m_{\beta}^{2} / M_{W}^{2}$ and remain negligible.

This implies that $\kappa^{\nu_{\tau} \nu_{\beta}}$ can be obtained from Eq. (2.24) for up-type fermions by simply replacing $u \rightarrow \nu_{\beta}$. By comparing the corresponding Hamiltonians Eqs. (2.7) and (2.27) we easily see that the relevant relation is

$$
\kappa^{\nu_{\tau} \nu_{\beta}}=\frac{\Delta c_{V / A}^{\nu_{\tau} \nu_{\beta}}}{c_{V / A}^{\nu_{\beta}}}=2 \Delta c_{V / A}^{\nu_{\tau} \nu_{\beta}} .
$$

Inserting the explicit neutrino couplings we obtain

$$
\kappa^{\nu_{\tau} \nu_{\beta}}=\kappa^{\nu_{\beta} \nu_{\tau}}=-2 \frac{g^{2}}{(4 \pi)^{2}} \frac{m_{\tau}^{2}}{M_{W}^{2}}=-\epsilon,
$$

where $\epsilon$ was defined in Eq. (1.5).
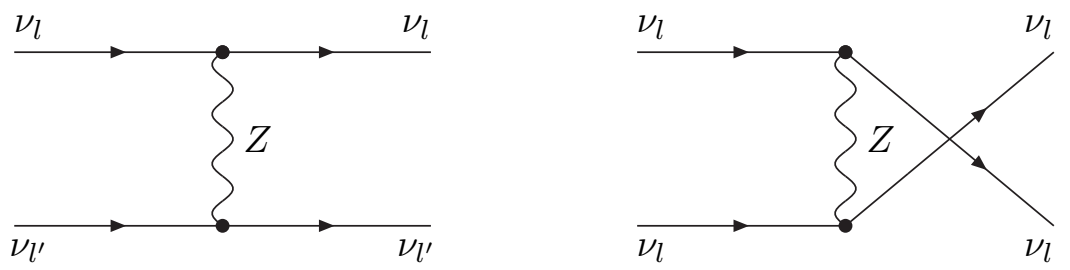

Figure 6: At tree level, neutrino forward scattering in a neutrino background proceeds through $t$-channel exchange of $Z$ bosons (left diagram), plus $u$-channel exchange in the case of identical flavours only (right diagram).

Finally we consider the equal-flavour corrections $\kappa^{\nu_{\tau} \nu_{\tau}}$ where the following differences must be taken into account:

- All scattering amplitudes are a factor two larger because in addition to the $t$-channel $Z$-exchange diagram that contributes to the $\nu_{l} \neq \nu_{l^{\prime}}$ interactions (left diagram in Fig. 6), also a $u$ channel opens (right diagram in Fig. 6). Moreover, due to a Fierz identity, the amplitudes of the $t$ - and $u$-channel diagrams are identical. In particular, the tree-level $\nu_{\tau}-\nu_{\tau}$ amplitude reads [cf. Eq. (2.11)]

$$
\mathcal{A}_{1}^{\nu_{\tau} \nu_{\tau}}=2 \times\left.\mathcal{A}_{1}^{\nu_{\tau} f}\right|_{f \rightarrow \nu_{\tau}}=\frac{-i g^{2}}{2 M_{W}^{2}} \mathcal{W}_{31} \mathcal{W}_{42}
$$


- In addition to the vertices and selfenergies of Figs. 2, 4 , similar diagrams must be included, that describe the correction to the $f \bar{f} Z$ vertex with $f=\nu_{\tau}$. In practice these two sets of "non-box" diagrams provide exactly the same correction. Including a further factor of two due to the presence of $u$-channel contributions we have [cf. Eq. (2.19)]

$$
\mathcal{A}_{234}^{\nu_{\tau} \nu_{\tau}}=2 \times 2 \times\left.\mathcal{A}_{234}^{\nu_{\tau} f}\right|_{f \rightarrow \nu_{\tau}}=\frac{i g^{4}}{(4 \pi)^{2}} \frac{m_{l}^{2}}{M_{W}^{4}}\left[3+\log \frac{m_{l}^{2}}{M_{W}^{2}}\right] \mathcal{W}_{31} \mathcal{W}_{42}
$$

- Finally the contribution of the box diagrams of Fig. 周 cannot be inferred from the calculation performed for the matter-background case since the terms of order $m_{f^{\prime}}^{2} / M_{W}^{2}$ cannot be neglected when $f=\nu_{l^{\prime}}=\nu_{\tau}$ and $f^{\prime}=l^{\prime}=\tau$. In this case, including again a factor two due to $u$-channel contributions, the ladder box diagram yields [cf. Eq. (2.21)]

$$
\mathcal{A}_{5}^{\nu_{\tau} \nu_{\tau}}=-\frac{1}{2} \frac{i g^{4}}{(4 \pi)^{2}} \frac{m_{\tau}^{2}}{M_{W}^{4}}\left[3+2 \log \left(\frac{m_{\tau}^{2}}{M_{W}^{2}}\right)\right] \mathcal{W}_{31} \mathcal{W}_{42} .
$$

Combining the above results we obtain

$$
\kappa^{\nu_{\tau} \nu_{\tau}}=-3 \frac{g^{2}}{(4 \pi)^{2}} \frac{m_{\tau}^{2}}{M_{W}^{2}}=-\frac{3}{2} \epsilon
$$

where $\epsilon$ was defined in Eq. (1.5).

In the context of neutrino oscillations (see Sec. 3) it proves useful to rewrite our results in a more compact notation:

$$
\mathcal{H}_{\nu \nu}=\frac{G_{\mathrm{F}}}{\sqrt{2}}\left\{\left[\bar{\nu}(1-\epsilon \mathrm{T}) \gamma^{\lambda} \omega_{L} \nu\right]\left[\bar{\nu}(1-\epsilon \mathrm{T}) \gamma_{\lambda} \omega_{L} \nu\right]+\frac{\epsilon}{2}\left[\bar{\nu} \mathbf{T} \gamma^{\lambda} \omega_{L} \nu\right]\left[\bar{\nu} \mathrm{T} \gamma_{\lambda} \omega_{L} \nu\right]\right\}
$$

where $\nu$ is now a vector in flavor space and in the weak-interaction basis

$$
\mathbf{T}=\operatorname{diag}(0,0,1)
$$

i.e. the matrix $\mathrm{T}$ is a projector on the $\tau$ direction in flavour space. Equation (2.36) holds at leading order in $\epsilon$. Note that the renormalization of the $\nu_{\tau}-\nu_{\tau}$ coupling implied by the first term in Eq. (2.36) is $1-2 \epsilon$ and has to be summed to the second term to provide the correct result $1-3 \epsilon / 2$. In other words, not only is the original $U(3)$ symmetry of the tree-level Hamiltonian Eq. (2.26) broken, but a simple description by renormalizing the coefficients $c_{V / A}^{\nu}$ is not possible.

For comparison with the refractive energy shifts caused by a background of charged fermions, we recall that at tree level a neutrino $\nu_{l}$ in a homogeneous and isotropic bath of $\nu_{l^{\prime}}$ experiences

$$
V_{\nu_{l} \nu_{l^{\prime}}}=\sqrt{2} G_{\mathrm{F}}\left(1+\delta_{l l^{\prime}}\right) n_{\nu_{l^{\prime}}} .
$$

Of course, the usual ultra-relativistic approximation was used where neutrinos are purely left-handed and $n_{\nu_{l^{\prime}}}$ is once more a net density of neutrinos minus antineutrinos. If neither 
$l$ nor $l^{\prime}$ are the $\tau$ flavour, radiative corrections are negligible at our level of approximation. For $l=\tau$ and $l^{\prime}=\beta \neq \tau$ our results imply

$$
V_{\nu_{\tau} \nu_{\beta}}=\sqrt{2} G_{\mathrm{F}}(1-\epsilon) n_{\nu_{\beta}}
$$

and analogously under the exchange of $\tau \leftrightarrow \beta$. Finally, for $l=l^{\prime}=\tau$ we find

$$
V_{\nu_{\tau} \nu_{\tau}}=\sqrt{2} G_{\mathrm{F}}(2-3 \epsilon) n_{\nu_{\tau}} \text {. }
$$

However, in the context of neutrino oscillations these energy shifts do not provide a useful understanding of the role of neutrino-neutrino refraction because the background neutrinos themselves are, in general, coherent superpositions of weak interaction states.

\section{Including radiative corrections in the oscillation equations}

To see how neutrino-neutrino refraction influences flavour oscillations it is most economical to represent the ensemble by flavour-space matrices of occupation numbers $\varrho_{i j}(\mathbf{p})=$ $\left\langle a_{i}^{\dagger}(\mathbf{p}) a_{j}(\mathbf{p})\right\rangle$ where $i$ and $j$ are flavour indices, $a^{\dagger}$ and $a$ are creation and annihilation operators and $\langle\cdots\rangle$ is an expectation value for the ensemble [7, 17]. The diagonal elements of these flavour-space matrices are the usual occupation numbers, whereas the off-diagonal elements encode relative phases between the different flavour components. The simultaneous treatment of neutrinos and antineutrinos becomes particularly transparent if one defines $\bar{\varrho}_{i j}(\mathbf{p})=\left\langle\bar{a}_{j}^{\dagger}(\mathbf{p}) \bar{a}_{i}(\mathbf{p})\right\rangle$ with "crossed" flavour indices where all over-barred quantities refer to antiparticles [7]. The equations of motion (EOM) for the flavour evolution are

$$
\mathrm{i} \varrho_{\mathbf{p}}=\left[\Omega_{\mathbf{p}}, \varrho_{\mathbf{p}}\right] \text { and } \mathrm{i} \dot{\bar{\varrho}}_{\mathbf{p}}=\left[\bar{\Omega}_{\mathbf{p}}, \bar{\varrho}_{\mathbf{p}}\right] \text {, }
$$

where $[\cdot, \cdot]$ is a commutator and the "matrices of oscillation frequencies" $\Omega_{\mathbf{p}}$ and $\bar{\Omega}_{\mathbf{p}}$ play the role of Hamiltonian operators in flavour space for the evolution of mode $\mathbf{p}$.

In an isotropic ensemble, vacuum masses, matter, and neutrinos provide at tree level the usual terms [7]

$$
\Omega_{\mathbf{p}}=+\frac{\mathrm{M}^{2}}{2 E}+\sqrt{2} G_{\mathrm{F}}\left(\mathrm{N}_{l}+\mathrm{N}_{\nu}\right), \quad \bar{\Omega}_{\mathbf{p}}=-\frac{\mathrm{M}^{2}}{2 E}+\sqrt{2} G_{\mathrm{F}}\left(\mathrm{N}_{l}+\mathrm{N}_{\nu}\right),
$$

where $\mathrm{M}^{2}$ is the neutrino mass-squared matrix and $E=|\mathbf{p}|$ for ultrarelativistic neutrinos. In the weak interaction basis, $\mathbf{M}^{2}=U D U^{\dagger}$ where $D=\operatorname{diag}\left(m_{1}^{2}, m_{2}^{2}, m_{3}^{2}\right)$ and $U$ is the usual mixing matrix. The matrix of number densities $\mathrm{N}_{l}$ for charged leptons is defined in the weak-interaction basis with diagonal elements $n_{l}=n_{l^{-}}-n_{l^{+}}$. The corresponding neutrino matrix is

$$
\mathrm{N}_{\nu}=\int \frac{\mathrm{d}^{3} \mathbf{q}}{(2 \pi)^{3}}\left(\varrho_{\mathbf{q}}-\bar{\varrho}_{\mathbf{q}}\right)
$$

If the medium is not isotropic, as in the case for neutrinos streaming from a SN core, the usual factor $\left(1-\mathbf{v}_{\mathbf{q}} \cdot \mathbf{v}_{\mathbf{p}}\right)$ must be included under the integral and the matrix $\mathrm{N}_{\nu}$ then also depends on p. This structure is identical in all that follows, so without loss of generality we can study the radiative modification assuming an isotropic medium. 
To see how our radiative corrections modify the structure of $\mathrm{N}_{\nu}$ it is easiest to begin with a hypothetical case of non-universal neutrino interactions of the form

$$
\mathcal{H}_{\mathrm{G}}=\frac{G_{\mathrm{F}}}{\sqrt{2}}\left(\bar{\nu} \mathrm{G} \gamma^{\lambda} \omega_{L} \nu\right)\left(\bar{\nu} \mathrm{G} \gamma_{\lambda} \omega_{L} \nu\right)
$$

where $G$ is a matrix in flavour space of dimensionless coupling constants. The tree-level standard-model case is $G=1$ (unit matrix in flavour space). It was previously shown that the oscillation equation is to be modified in the form [7]

$$
\mathrm{N}_{\nu} \rightarrow \mathrm{N}_{\nu}^{\mathrm{eff}}(\mathrm{G})=\int \frac{\mathrm{d}^{3} \mathbf{q}}{(2 \pi)^{3}}\left\{\mathrm{G}\left(\varrho_{\mathbf{q}}-\bar{\varrho}_{\mathbf{q}}\right) \mathrm{G}+\mathrm{G} \operatorname{Tr}\left[\left(\varrho_{\mathbf{q}}-\bar{\varrho}_{\mathbf{q}}\right) \mathrm{G}\right]\right\}
$$

For $\mathrm{G}=1$ this expression is identical to $\mathrm{N}_{\nu}$ up to an additive term that is proportional to the unit matrix and to the net total neutrino density

$$
n_{\nu}=\int \frac{\mathrm{d}^{3} \mathbf{q}}{(2 \pi)^{3}} \operatorname{Tr}\left(\varrho_{\mathbf{q}}-\bar{\varrho}_{\mathbf{q}}\right) .
$$

This quantity is conserved under oscillations, so $\mathrm{N}_{\nu}$ and $\mathrm{N}_{\nu}^{\mathrm{eff}}(1)$ play identical roles and actually we could subtract Eq. (3.6) from Eq. (3.5) to achieve $\mathrm{N}_{\nu}=\mathrm{N}_{\nu}^{\mathrm{eff}}(1)$.

Our calculation of the radiative correction of neutrino-neutrino interactions reveals that the flavour-sensitive difference of the scattering amplitudes can be expressed, at leading order and in the "large $m_{\tau}$ limit," in the form of Eq. (2.36). Therefore, radiative corrections effectively enter with two different matrices of non-universal couplings. In the oscillation equation we thus need to substitute

$$
\mathrm{N}_{\nu} \rightarrow \mathrm{N}_{\nu}^{\mathrm{eff}}(1-\epsilon \mathrm{T})+\frac{\epsilon}{2} \mathrm{~N}_{\nu}^{\mathrm{eff}}(\mathrm{T})
$$

where the projection matrix $\mathrm{T}$ was defined in Eq. (2.37). In the weak-interaction basis this is explicitly to leading order in $\epsilon$

$$
\mathrm{N}_{\nu} \rightarrow \mathrm{N}_{\nu}-\epsilon\left(\begin{array}{ccc}
0 & 0 & \mathrm{~N}_{\nu}^{e \tau} \\
0 & 0 & \mathrm{~N}_{\nu}^{\mu \tau} \\
\mathrm{N}_{\nu}^{\tau e} & \mathrm{~N}_{\nu}^{\tau \mu} & \mathrm{N}_{\nu}^{e e}+\mathrm{N}_{\nu}^{\mu \mu}+2 \mathrm{~N}_{\nu}^{\tau \tau}
\end{array}\right)
$$

where we have neglected a term proportional to the unit matrix that is irrelevant for oscillations.

\section{Conclusions}

We have calculated the flavour non-universal correction for neutrino-neutrino refraction in the framework of the Standard Model. The dominant term arises at loop level and involves $\tau$ leptons circulating in the loop. In the course of our derivation we have reproduced a similar term for a background medium of charged fermions that had been calculated previously by Botella, Lim and Marciano [3]. One novel feature of our result is that the radiatively corrected effective neutrino-neutrino Hamiltonian can not be expressed in terms 
of renormalized tree-level coupling constants. This different structure derives from the box diagrams with the exchange of two $\mathrm{W}$ bosons.

The impact of neutrino-neutrino refraction on collective neutrino oscillations is not easily assessed by comparing refractive energy shifts relative to those caused by an ordinarymatter background. The recent torrent of activities concerning collective SN neutrino oscillations was essentially triggered by the insight that the nonlinear nature of the equations allows for large collective effects even if the ordinary-matter background causes larger energy shifts than the neutrino background. This is traced back to the phenomenon that the refractive-index matrix caused by ordinary matter and by background neutrinos are usually not diagonal in the same basis because of neutrino flavor oscillations.

Our radiative corrections provide non-universal neutrino-neutrino interactions similar to, but not identical with, the case of non-standard interactions studied in Ref. [18], suggesting the possibility of interesting collective oscillation phenomena. However, a realistic assessment requires a dedicated study including the impact of a dense ordinary-matter background and multi-angle effects.

We also note that in certain supersymmetric scenarios the neutrino-fermion radiative corrections could be enhanced up to two order of magnitudes with respect to the standard case 19, 20]. Similar enhancements might be expected for neutrino-neutrino refraction.

\section{Acknowledgments}

In Munich, we acknowledge partial support by the Deutsche Forschungsgemeinschaft under grant TR-27 "Neutrinos and Beyond" and the Cluster of Excellence "Origin and Structure of the Universe." We thank B. Dasgupta and J. Gava for comments. The work of AM is supported by the Italian Istituto Nazionale di Fisica Nucleare (INFN). P.S. thanks the Galileo Galilei Institute for Theoretical Physics for hospitality and the INFN for partial support during the initial stage of this work. A.M. thanks CERN for kind hospitality during the initial development of this project.

\section{References}

[1] L. Wolfenstein, "Neutrino oscillations in matter," Phys. Rev. D 17, 2369 (1978).

[2] S. P. Mikheev and A. Yu. Smirnov, "Resonance enhancement of oscillations in matter and solar neutrino spectroscopy," Yad. Fiz. 42, 1441 (1985) [Sov. J. Nucl. Phys. 42, 913 (1985)].

[3] F. J. Botella, C. S. Lim and W. J. Marciano, "Radiative corrections to neutrino indices of refraction," Phys. Rev. D 35, 896 (1987).

[4] A. S. Dighe and A. Y. Smirnov, "Identifying the neutrino mass spectrum from the neutrino burst from a supernova," Phys. Rev. D 62, 033007 (2000) [hep-ph/9907423].

[5] E. K. Akhmedov, C. Lunardini and A. Y. Smirnov, "Supernova neutrinos: Difference of $\nu_{\mu}-\nu_{\tau}$ fluxes and conversion effects," Nucl. Phys. B 643, 339 (2002) [hep-ph/0204091].

[6] J. T. Pantaleone, "Neutrino oscillations at high densities," Phys. Lett. B 287, 128 (1992).

[7] G. Sigl and G. Raffelt, "General kinetic description of relativistic mixed neutrinos," Nucl. Phys. B 406, 423 (1993). 
[8] V. A. Kostelecky and S. Samuel, "Selfmaintained coherent oscillations in dense neutrino gases," Phys. Rev. D 52, 621 (1995). [hep-ph/9506262].

[9] S. Pastor, G. G. Raffelt and D. V. Semikoz, "Physics of synchronized neutrino oscillations caused by self-interactions," Phys. Rev. D 65, 053011 (2002). [hep-ph/0109035].

[10] H. Duan and J. P. Kneller, "Neutrino flavor transformation in supernovae," arXiv:0904.0974 [astro-ph.HE].

[11] A. Esteban-Pretel, S. Pastor, R. Tomàs, G. G. Raffelt and G. Sigl, "Mu-tau neutrino refraction and collective three-flavor transformations in supernovae," Phys. Rev. D 77, 065024 (2008) [arXiv:0712.1137].

[12] J. Gava and C. Volpe, "Collective neutrinos oscillation in matter and CP-violation," Phys. Rev. D 78, 083007 (2008) [arXiv:0807.3418].

[13] A. Esteban-Pretel, A. Mirizzi, S. Pastor, R. Tomàs, G. G. Raffelt, P. D. Serpico and G. Sigl, "Role of dense matter in collective supernova neutrino transformations," Phys. Rev. D 78, 085012 (2008) [arXiv:0807.0659].

[14] G. G. Raffelt, "Stars As Laboratories For Fundamental Physics: The Astrophysics Of Neutrinos, Axions, And Other Weakly Interacting Particles," Chicago, USA: Univ. Pr. (1996) $664 \mathrm{p}$.

[15] M. Fukugita and T. Yanagida, "Physics of neutrinos and applications to astrophysics," Berlin, Germany - Springer (2003) 593 p.

[16] C. Giunti, C. W. Kim and W. P. Lam, "Radiative decay and magnetic moment of neutrinos in matter," Phys. Rev. D 43, 164 (1991).

[17] A. D. Dolgov, "Neutrinos in the early universe," Yad. Fiz. 33 (1981) 1309 [Sov. J. Nucl. Phys. 33, 700 (1981)].

[18] M. Blennow, A. Mirizzi and P. D. Serpico, "Nonstandard neutrino-neutrino refractive effects in dense neutrino gases," Phys. Rev. D 78, 113004 (2008) [arXiv: 0810.2297].

[19] E. Roulet, "Supersymmetric radiative corrections to neutrino indices of refraction," Phys. Lett. B 356, 264 (1995) [hep-ph/9506221].

[20] J. Gava and C. C. Jean-Louis, "SUSY radiative corrections on mu-tau neutrino refraction including possible R-parity breaking interactions," arXiv:0907.3947 [hep-ph]. 